\title{
Molecular and morphological characterization of pomegranate (Punica granatum L.) genotypes sampled from Coruh Valley in Turkey
}

\author{
E. Orhan ${ }^{1}$, S. Ercisli ${ }^{2}$, A. Esitken ${ }^{3}$ and M. Sengul ${ }^{4}$ \\ ${ }^{1}$ Department of Horticulture, Faculty of Agriculture, Ataturk University, \\ Erzurum, Turkey \\ ${ }^{2}$ Department of Agricultural Biotechnology, Faculty of Agriculture, \\ Ataturk University, Erzurum, Turkey \\ ${ }^{3}$ Department of Horticulture, Faculty of Agriculture, Selcuk University, \\ Selcuklu, Konya, Turkey \\ ${ }^{4}$ Department of Food Engineering, Faculty of Agriculture, Ataturk University, \\ Erzurum, Turkey \\ Corresponding author: S. Ercisli \\ E-mail: sercisli@gmail.com
}

Genet. Mol. Res. 13 (3): 6375-6382 (2014)

Received June 17, 2013

Accepted October 10, 2013

Published January 14, 2014

DOI http://dx.doi.org/10.4238/2014.January.14.4

\begin{abstract}
The pomegranate is one of the oldest fruits that are traditionally consumed by the local inhabitants of the Coruh Valley, Turkey. In this study, the molecular and morphological characteristics of 19 promising pomegranate genotypes selected from the Coruh Valley were evaluated. For the morphological evaluation, 22 quantitative fruit characteristics were used. For the molecular evaluation, 47 random amplified polymorphic DNA (RAPD) primers were used for polymerase chain reaction analysis. The principle component analysis of 22 quantitative fruit characteristics revealed that fruit weight and skin color are dominant traits for genotype discrimination. The unweighted average distance cluster of fruit characteristics revealed 3 distinct groups. Among the 47 RAPD primers, 9 exhibited reliable polymorphic patterns, and generated a total of
\end{abstract}


63 RAPD bands, of which $49.2 \%$ were polymorphic. The similarity matrix showed that the highest (0.920) and lowest (0.556) genetic similarities occurred between the APS13 and APS28 genotypes and the APS12 and APS42 genotypes, respectively. We determined clear discrepancies between the morphological and molecular data; consequently, the differences obtained among genotypes for fruit characteristics did not support genetic relationships among genotypes. In conclusion, molecular data provided the most reliable results at the DNA level.

Key words: Punica granatum; Molecular characterization; RAPD; Pomegranate

\section{INTRODUCTION}

Pomegranate is considered an excellent tree for growing in arid or semiarid zones because of its resistance to drought conditions. It is widely cultivated in countries in the Mediterranean region, including Egypt, Morocco, Spain, Tunisia, and Turkey. Large numbers of scattered pomegranate trees located on the borders or within other fruit orchards have been reported in many Mediterranean countries, with the fruit being very popular in local markets. Pomegranate is cultivated in India and Iran, and to some extent the USA (California), Russia, China, and Japan, for fruit production, and it is also grown as an ornamental tree in East Asia (Mars and Marrakchi, 1999).

Turkey is one of the native countries of pomegranate, with this plant being primarily cultivated in the Aegean part of the Mediterranean, southern Anatolia, northeast part of Turkey since ancient times (Ercisli, 2004). The fresh juice of pomegranate contains 85\% moisture, and considerable amounts of total soluble solids (TSS), total sugars, reducing sugars, anthocyanins, phenolics, ascorbic acid (vitamin C), and proteins (El-Nemr et al., 1990), and has also been reported to be a rich source of antioxidants (Kulkarni and Aradhya, 2005).

Previous classifications and evaluations of the pomegranate have primarily focused on the phenotypic expressions of pomegranate, such as color, shape, and other agronomical characterisctics of the fruit (Ercan et al., 1992; Polat et al., 1999; Ozkan, 2005). However, information about these environmentally influenced morphological and phenotypic characteristics are not sufficient to identify pomegranate genotypes, because of the environmental plasticity of these traits. Thus, environmentally free genotypic traits must be identified for the proper identification and estimation of genetic diversity among these genotypes.

Several molecular methods are used to document the genetic information of plants (Briard et al., 2001). The RAPD technique employs 10 bp random primers to locate random segments of genomic DNA, and reveal polymorphisms. RAPD analysis provides a simple and reliable method for measuring genomic variation in plants (Taskin et al., 2012; Poyraz et al., 2012; Surgun et al., 2012; Yaycili and Alikamanoglu, 2012). RAPD is a relatively straightforward technique to apply, in which an unlimited number of loci may potentially be examined. Therefore, RAPD analysis has a number of advantages over restriction fragment length polymorphism (RFLP) and other techniques (Lynch and Milligan, 1994). Another advantage of RAPD over other genetic documentation systems is that it has a universal set of primers; hence, preliminary work is not required, such as probe isolation, filter preparation, or nucleotide sequencing (Williams et al., 1990). The ease and simplicity of the RAPD technique makes it 
ideal for genetic mapping, plant, and animal breeding programs, and DNA fingerprinting, with particular utility in the field of population genetics. In many instance, only a small number of primers are required to identify polymorphism within species (Williams et al., 1990).

Limited comparable morphological and molecular studies on the genetic relationships and diversity of pomegranate have been published. The selected genotypes show many promising agronomic traits, including high yield capacity, pest and disease resistance, and promising fruit characteristics. Crosses between these genotypes could generate new and improved pomegranate varieties. In this study, we report the relationships of molecular and morphological characteristics of 19 pomegranate genotypes from Turkey.

\section{MATERIAL AND METHODS}

\section{Plant material}

The leaves and fruit of 19 selected pomegranate (Punica granatum L.) genotypes were sampled from Coruh Valley, Turkey. The coordinates and altitude of the region are $40^{\circ} 49^{\prime} 24^{\prime \prime} \mathrm{N}$ $41^{\circ} 32^{\prime} 55^{\prime \prime} \mathrm{E}$ and $505 \mathrm{~m}$ altitude.

\section{Morphological characterization}

For the morphological characterization, mature fruits were randomly harvested from the trees, and then transported to the laboratory for analysis. Twenty-two quantitative fruit characteristics were determined, including fruit weight, fruit width, fruit length, peel thickness, peel weight, aril length, aril width, aril thickness, aril weight, juice yield, soluble solids, dry matter, $\mathrm{pH}$, acidity, maturity index, skin color L, skin color a, skin color b, aril color L, aril color a, aril color b, and vitamin C.

\section{DNA extraction}

For the molecular characterization, 20 leaves from the top of 90-day-old primary branches per genotype were collected separately and immediately stored at $-80^{\circ} \mathrm{C}$ for DNA extraction. Genomic DNA was extracted from powdered leaf materials using method modified from Lin et al. (2001). The purity and quantity of genomic DNA was determined spectrophotometrically, and confirmed against known concentrations of unrestricted lambda DNA by electrophoresis on $0.8 \%$ agarose gel.

\section{RAPDs}

Forty-seven primers (Operon Technologies Inc., Alameda, CA, USA) were used to generate the RAPD profiles. PCR amplification reactions were carried out in $30 \mu 1$ final volume of reaction mixture containing 3.0 $\mu \mathrm{L} \mathrm{10X} \mathrm{Buffer,} 1.2 \mu \mathrm{L} 10 \mathrm{mM}$ dNTPs, $1.2 \mu \mathrm{L} 25 \mathrm{mM}$ magnesium chloride, $2.0 \mu \mathrm{L} 5 \mu \mathrm{M}$ primer, $0.4 \mu \mathrm{L} 5 \mathrm{U}$ Taq polymerase, $19.2 \mu \mathrm{L}$ water, and $3.0 \mu \mathrm{L} 100$ $\mathrm{ng} / \mu \mathrm{L}$ sample DNA. The thermalcycler (Eppendorf Company, Westbury, NY, USA) was programmed to $2 \mathrm{~min}$ at $95^{\circ} \mathrm{C} ; 2$ cycles of $30 \mathrm{~s}$ at $95^{\circ} \mathrm{C}, 1 \mathrm{~min}$ at $37^{\circ} \mathrm{C}$, and $2 \mathrm{~min}$ at $72^{\circ} \mathrm{C} ; 2$ cycles of $30 \mathrm{~s}$ at $95^{\circ} \mathrm{C}, 1 \mathrm{~min}$ at $35^{\circ} \mathrm{C}$, and $2 \mathrm{~min}$ at $72^{\circ} \mathrm{C} ; 41$ cycles of $30 \mathrm{~s}$ at $94^{\circ} \mathrm{C}, 1 \mathrm{~min}$ at $35^{\circ} \mathrm{C}$, and $2 \mathrm{~min}$ at $72^{\circ} \mathrm{C}$; followed by a final $5 \mathrm{~min}$ extension at $72^{\circ} \mathrm{C}$, and then brought down to $4^{\circ} \mathrm{C}$. 


\section{Electrophoresis}

The PCR products $(27 \mu \mathrm{L})$ were mixed with $6 \mathrm{X} 3 \mu \mathrm{L}$ gel loading buffer and loaded on agarose $(1.5 \% \mathrm{w} / \mathrm{v})$ gel electrophoresis in $0.5 \mathrm{X}$ TBE (Tris-Borate-EDTA) buffer at $70 \mathrm{~V}$ for $150 \mathrm{~min}$. The gel was stained in ethidium bromide solution ( $2 \mu \mathrm{L} \mathrm{Etbr} / 100 \mathrm{~mL} 1 \mathrm{X}$ TBE buffer $)$ for $40 \mathrm{~min}$, and visualized under UV by the Bio Doc Image Analysis System with the Uvisoft analysis package (Cambridge, UK).

\section{Data analysis}

Fruit characteristic data were analyzed using SAS procedures. The means and standard deviations were calculated using PROC TABULATE. PROC PRINCOMP was used to conduct principle component (PCo) analysis, using 22 quantitative fruit characteristics. For the molecular analysis, each gel was analyzed by scoring the presence (1) or absence (0) of polymorphic bands in individual lanes. The NTSYS-pc software ver. 2.02 (Roholf, 1998) was used to estimate genetic similarities with the Jaccard's coefficient. The matrix of generated similarities was analyzed by the unweighted pairgroup method with arithmetic average (UPGMA), using the SAHN clustering module. The cophenetic module was applied to compute a cophenetic value matrix using the UPGMA matrix. The MXCOMP module was then used to compute the cophenetic correlation, i.e., to test the goodness of fit of the cluster analysis to the similarity matrix.

\section{RESULTS}

\section{Morphological data}

We observed considerable morphological variation for almost all fruit characteristics among the searched genotypes, with certain important fruit characteristics being shown in Table 1. For example, fruit weight ranged from $218 \mathrm{~g}$ (APS36) to $540 \mathrm{~g}$ (APS13), indicating that the APS13 genotype has the heaviest fruits. We also detected broad variation for skin color a values (green to red; 3.21-70.03) and skin color b values (5.97-52.66).

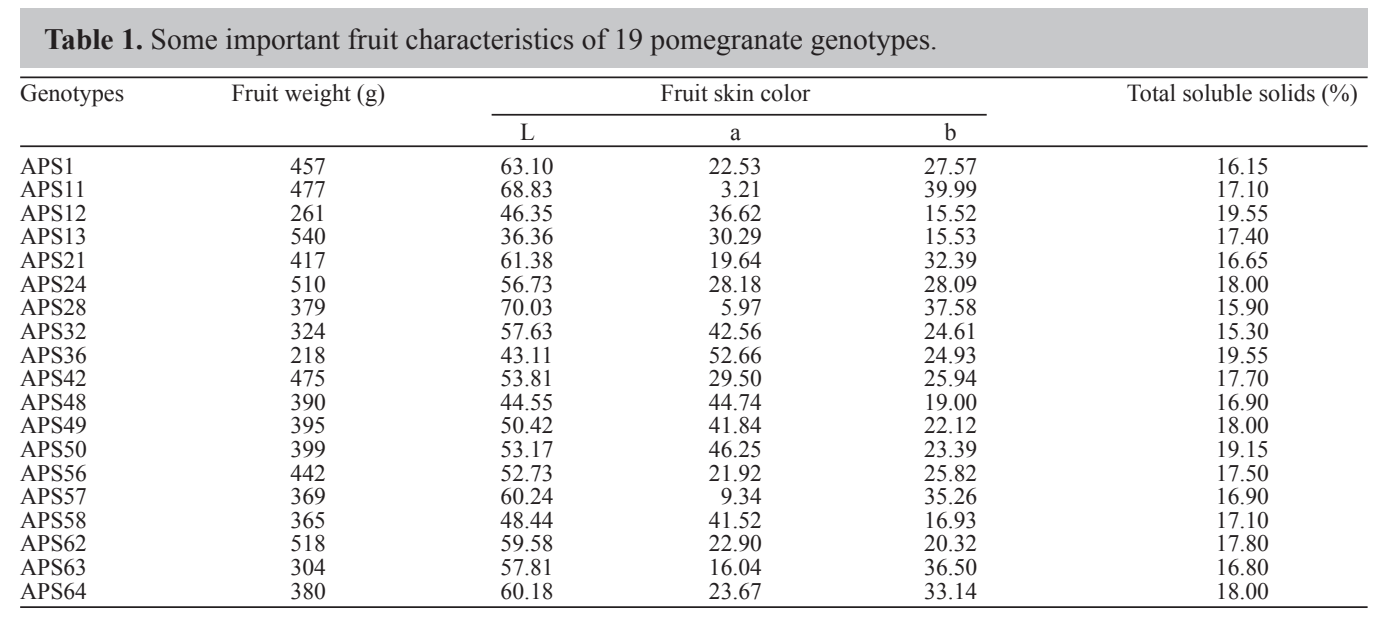


The principle component analysis results are shown in Table 2. The first 3 PCs explained virtually all of the morphological variation among the tested genotypes. PC1 contained $85 \%$ the variation, followed by 10 and $5 \%$ for PCs 2 and 3, respectively (Table 2 ). The highest correlation among the variables and PC1 was for fruit weight.

Table 2. Coefficients and eigenvalues for the first three principle components (PC) of PCA for 19 pomegranate genotypes using 22 quantitative fruit characteristics.

\begin{tabular}{lccc}
\hline Variable & PC1 & PC2 & PC3 \\
\hline Fruit weight & 0.81 & 0.01 & 0.18 \\
Fruit width & 0.05 & 0.02 & 0.01 \\
Fruit length & 0.02 & 0.02 & 0.02 \\
Peel thickness & 0.01 & 0.04 & 0.02 \\
Peel weight & 0.01 & 0.01 & 0.01 \\
Aril length & 0.00 & 0.00 & 0.01 \\
Aril width & 0.01 & 0.02 & 0.00 \\
Aril thickness & -0.04 & -0.11 & -0.01 \\
Aril weight & 0.08 & 0.02 & 0.12 \\
Juice yield & 0.04 & 0.02 & 0.02 \\
Soluble solids & 0.00 & 0.01 & -0.01 \\
Dry matter & 0.01 & 0.00 & -0.02 \\
pH & 0.00 & 0.01 & 0.00 \\
Acidity & 0.00 & -0.04 & -0.01 \\
Maturity index & 0.05 & -0.12 & 0.23 \\
Skin color L & -0.03 & 0.18 & -0.11 \\
Skin color a & -0.01 & -0.12 & 0.09 \\
Skin color b & 0.01 & -0.05 & -0.03 \\
Aril color L & 0.02 & 0.05 & -0.02 \\
Aril color a & 0.04 & -0.02 & 0.09 \\
Aril color b & 0.02 & -0.01 & -0.02 \\
Vitamin C & 0.01 & 1882 & -0.01 \\
Eigenvalue & 30112 & 407 & 698 \\
Difference & 27112 & 0.10 & 904 \\
Proportion & 0.85 & 0.93 & 0.05 \\
Cumulative & 0.87 & 1.00 \\
\hline
\end{tabular}

The UPGMA phenogram of the data obtained from 22 quantitative fruit characteristics is presented in Figure 1. The phenogram produced 3 distinct clusters. The first cluster contained 'APS12', 'APS24', and 'APS36', which have different fruit characteristics to each other. The second cluster contained 'APS64', 'APS49', 'APS57', 'APS32', and 'APS62' genotypes. The third cluster contained 11 genotypes, which also have different fruit characteristics, and were further grouped into 3 sub-clusters.

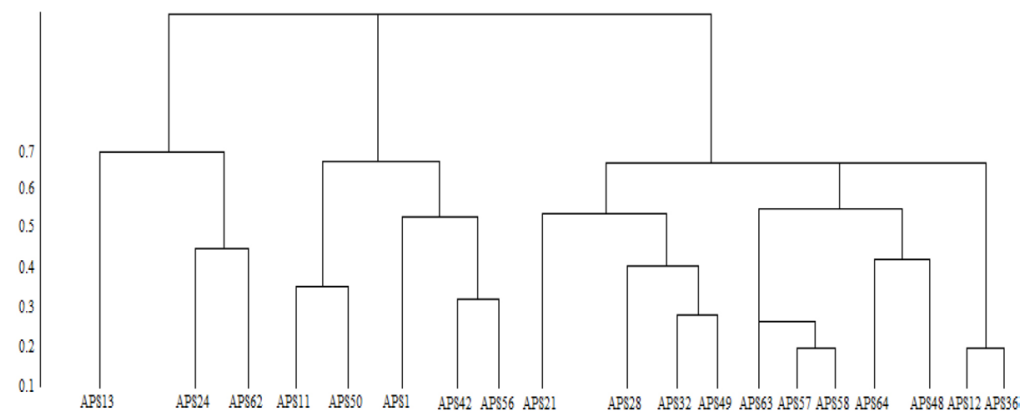

Figure 1. UPGMA phenogram of 19 pomegranate genotypes based on average distances among cultivar means of 22 quantitative fruit characteristics. 


\section{Molecular (RAPD) data}

The results of the RAPD analysis are summarized in Table 3. A total of 47 decamer oligonucleotide primers were used to investigate 19 selected pomegranate genotypes all belonging to $P$. granatum. In total, 9 out of the 47 primers produced good and reproducible polymorphic bands among the 19 pomegranate genotypes, and were used for further analysis. The 9 random primers generated a total of 63 RAPD bands, of which 31 were polymorphic (Table 3). The highest polymorphism $(80.0 \%)$ was observed for the OPBB-3 primer, while the lowest polymorphism (33.3\%) was observed for the OPBB-4 and OPBC-8 primers (Table 3). The size of the amplified fragments ranged from 200 to $2000 \mathrm{bp}$ for all primers. The similarity matrix showed that the highest $(0.920)$ and lowest (0.556) genetic similarities occurred between the APS13 and APS28 genotypes and the APS12 and APS42 genotypes, respectively.

\begin{tabular}{|c|c|c|c|c|c|}
\hline Primer code & Sequence $5^{\prime}$ to $3^{\prime}$ & Size (bp) min-max & Total band No. & Polymorphic bands & Polymorphism ratio (\%) \\
\hline OPBA-06 & GGACGACCGT & $480-2000$ & 7 & 3 & 42.9 \\
\hline OPBB-03 & TCACGTGGCT & $470-1200$ & 5 & 4 & 80.0 \\
\hline OPBB-04 & ACCAGGTCAC & $250-1400$ & 6 & 2 & 33.3 \\
\hline OPBB-07 & GAAGGCTGGG & $450-2000$ & 6 & 4 & 66.7 \\
\hline OPBB-08 & TCGTCGAAGG & $280-1200$ & 8 & 6 & 75.0 \\
\hline OPBB-10 & ACTTGCCTGG & $350-900$ & 5 & 2 & 40.0 \\
\hline OPBD-07 & GAGCTGGTCC & $200-950$ & 8 & 3 & 37.5 \\
\hline OPBD-17 & GTTCGCTCCC & $250-1100$ & 9 & 4 & 44.4 \\
\hline OPBC-08 & GGTCTTCCCT & $250-2000$ & 9 & 3 & 33.3 \\
\hline Total & - & - & 63 & 31 & - \\
\hline Average & - & $200-2000$ & - & 31 & 49.2 \\
\hline
\end{tabular}

The dendrogram produced from the RAPD markers grouped the genotypes into 2 main clusters, which contained 2 and 17 genotypes, respectively (Figure 2). Cluster 1 contained the APS12 and APS-49 pomegranate genotypes. Cluster 2 was divided into 2 subclusters, with subcluster 1 containing just 1 genotype (APS-36), while subcluster 2 contained the remaining 16 genotypes.

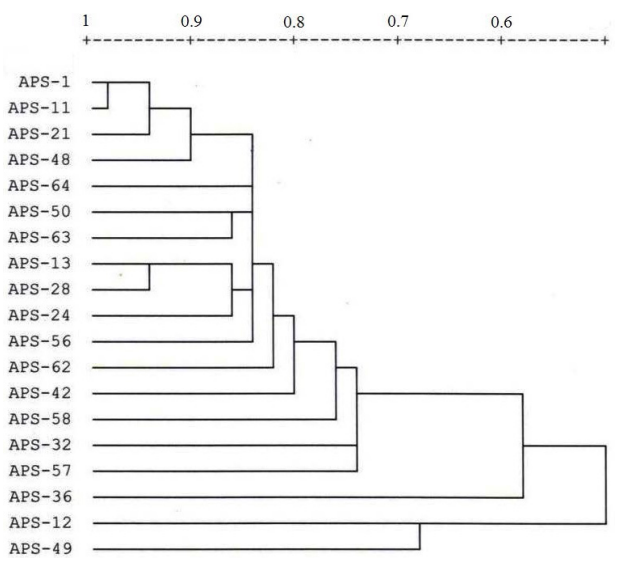

Figure 2. UPGMA dendrogram of 19 pomegranate genotypes based on 17 RAPD primers. 


\section{DISCUSSION}

Previous studies in Turkey recorded highly variable fruit size in pomegranate cultivars, ranging from 213 to $610 \mathrm{~g}$ (Durgaç et al., 2008). Principle component analyses were previously employed by several researchers to characterize pomegranate germplasm. For example, Mars and Marrakchi (1999) studied 30 pomegranate accessions in Tunisia, and found that the discriminating characteristics of the germplasms were fruit size, color, and juice characteristics. The same traits were found to be important in the study of Drogoudi et al. (2005), in which the authors determined several characteristics of 20 Greek pomegranate cultivars. Therefore, the discriminating traits obtained from the PCo results of the current study supported these previous studies.

Based on the molecular results, the genotypes APS-13 and APS-28 are genetically closer compared to all other studied genotypes. In addition, the low genetic similarity that was observed between the APS-12 and APS-42 genotypes indicates their utility for use in heterosis breeding. The average genetic similarity among the genotypes was 0.613 , which confirms the presence of significant genetic diversity among the selected genotypes. Hence, these genotypes should be preserved as valuable genetic resources for breeding. Earlier studies of pomegranate using RAPD also recorded large genetic variations (22-85\%) among cultivars (Sarkhosh et al., 2006; Ercisli et al., 2007; Zamani et al., 2007; Durgaç et al., 2008).

The high genetic distances observed among these genotypes clearly indicate that they may have originated from genetically divergent parents, or have a long history of adaptation to their respective micro-climatic regions. Moreover, continuous seed propagation for $1000 \mathrm{~s}$ of years, in combination with human selection on various traits, probably created a diversity of pomegranate genotypes. This genetic diversity is an important resource that could be used to enhance pomegranate breeding programs in various ways.

This study presents one of the first attempts to use molecular markers to investigate the genetic relationships of a wide number of pomegranate genotypes belonging to $P$. granatum grown in Turkey. The RAPD technique could prove a useful tool for the management of pomegranate and other fruit plant collections. This data provides a scientific basis for the future selection and management of pomegranate germplasm. The results of this study indicate that the level of polymorphism among pomegranate is appreciably high in Turkey, and that these genotypes could be used in breeding programs. In conclusion, the information generated in this study is useful for enhancing pomegranate crops through breeding.

\section{ACKNOWLEDGMENTS}

Research supported by the Turkish Scientific Council (TUBITAK) under the project number \#105O636. The authors would like to thank to TUBITAK for this financial support.

\section{REFERENCES}

Briard M, Le Clerc V, Mausset AE and Veret A (2001). A comparative study on the use of ISSR microsatellites and RAPD markers for varietal identification of carrot genotypes. Acta Hortic. 546: 377-385.

Drogoudi PD, Tsipouridis C and Michailidis Z (2005). Physical and chemical characteristics of pomegranates. HortScience 40: 1200-1203.

Durgaç C, Ozgen M, Simsek O, Kaçar YA, et al. (2008). Molecular and pomological diversity among pomegranate 
(Punica granatum L.) cultivars in Eastern Mediterranean region of Turkey. Afr. J. Biotechnol. 7: 1294-1301.

El-Nemr SE, Ismail IA and Ragab M (1990). Chemical composition of juice and seeds of pomegranate fruit. Food Nahrung 34: 601-606.

Ercan N, Ozvardar S, Gonulsen N, Baldiran E, et al. (1992). Determination of Suitable Pomegranate Cultivars for Aegean Region. Proceedings of 1st National Horticultural Congress, Izmir, 553-557.

Ercisli S (2004). A short review of the fruit germplasm resources of Turkey. Genet. Res. Crop Evol. 51: 419-435.

Ercisli S, Agar G, Orhan E, Yildirim N, et al. (2007). Interspecific variability of RAPD and fatty acid composition of some pomegranate cultivars (Punica granatum L.) growing in Southern Anatolia Region in Turkey. Biochem. Systemat. Ecol. 35: 764-769.

Kulkarni AP and Aradhya SM (2005). Chemical changes and antioxidant activity in pomegranate arils during fruit development. Food Chem. 93: 319-324.

Lin RC, Ding ZS, Li LB and Kuang TY (2001). A rapid and efficient DNA minipreparation suitable for screening transgenic plants. Plant Mol. Biol. Rep. 19: 379.

Lynch M and Milligan BG (1994). Analysis of population genetic structure with RAPD markers. Mol. Ecol. 3: 91-99.

Mars M and Marrakchi M (1999). Diversity of pomegranate (Punica granatum L.) germplasm in Tunisia. Genet. Res. Crop Evol. 46: 461-467.

Ozkan Y (2005). Investigations on physical and chemical characteristics of some pomegranate genotypes (Punica granatum L.) of Tokat province in Turkey. Asian J. Chem. 17: 939-942.

Polat AA, Durgac C, Kamiloglu O, Mansuroglu M, et al. (1999). Studies on Determination of Pomological Characteristics of Some Pomegranate Types Grown in Kirikhan District of Hatay Province. Proceedings of 3rd National Horticultural Congress, Ankara, 746-750.

Poyraz IE, Sozen E, Ataslar E and Poyraz I (2012). Determination of genetic relationships among Velezia L. Caryophyllaceae species using RAPD markers. Turk. J. Biol. 36: 293-302.

Roholf FJ (1998). NTSYS-pc Numerical Taxonomy and Multivariate Analysis System. Version 2.00. Exeter Software, Setauket.

Sarkhosh A, Zamani Z, Fatahi R and Ebadi A (2006). RAPD markers reveal polymorphism among some Iranian pomegranate (Punica granatum L.) genotypes. Sci. Hortic. 111: 24-29.

Surgun Y, Col B and Burun B (2012). Genetic diversity and identification of some Turkish cotton genotypes (Gossypium hirsutum L.) by RAPD-PCR analysis. Turk. J. Biol. 36: 143-150.

Taskin BG, Vardareli N, Dogac E, Mammadov R, et al. (2012). Genetic diversity of natural Cyclamen alpinum populations. Turk. J. Biol. 36: 413-422.

Williams JG, Kubelik AR, Livak KJ, Rafalski JA, et al. (1990). DNA polymorphisms amplified by arbitrary primers are useful as genetic markers. Nucleic Acids Res. 18: 6531-6535.

Yaycili O and Alikamanoglu S (2012). Induction of salt-tolerant potato (Solanum tuberosum L.) mutants with gamma irradiation and characterization of genetic variations via RAPD-PCR analysis. Turk. J. Biol. 36: 405-412.

Zamani Z, Sarkhosh A, Fatahi R and Ebadi A (2007). Genetic relationships among pomegranate genotypes studied by fruit characteristics and RAPD markers. J. Hortic. Sci. Biotechnol. 82: 11-18. 